\section{Lupus pernio (Besnier-Tenneson syndrome): A rare form of sarcoidosis}

\author{
Amal Hubail, Roza Belkharoeva, \\ Natalya Tepluk, Tatyana Belerosova \\ Hospital of Skin and Venereal Disease \\ Hospital named after VA Rakhmanov. \\ I.M. Sechenov
}

First Moscow State Medical University, Moscow, Russia

\begin{abstract}
Lupus pernio (LP) is a chronic non-life threatining type of cutaneous sarcoidosis that can be related to chronic fibrotic sarcoidosis, hyperglobulinemia and hypercalcemia. The aim of this case report is to evaluate the clinical and demographic features of cutaneous sarcoidosis mainly presenting with a rare manifestation of LP. In this paper we report a case of systemic sarcoidosis presenting with LP and a review of the available literature. LP is a rare presentation with infiltrated erythematoviolaceous plaques affecting the nose. We address the main management approach, and possible association with an underlying systemic sarcoidosis. LP is a rare but chronic manifestation of systemic sarcoidosis that needs to be treated in order to prevent cosmetic defects and psychological effects. It is important to recognize such a condition early in order to avoid a delay in treatment and worsening of the condition, both physically and psychologically. Further research regarding the diagnostic approach and management is required to understand this condition thoroughly.
\end{abstract}

\section{Introduction}

Sarcoidosis is a multisystemic granulomatous condition characterized by collection of non-caseating epithelioid granulomas. It can affect the pulmonary, reticuloendothelial, skin, gastrointestinal, cardiac, musculoskeletal, endocrine, or central nervous system. Sarcoidosis can be divided into two broad catogeries: systemic or cutaneous. ${ }^{1}$

Lupus pernio (LP; Besnier-Tenneson syndrome $)^{2}$ is a form of cutaneous manifestation which is expressed as blue red to violet smooth shiny nodules and plaques on the head and neck, predominantly on the nose, ears, lips, and cheeks. ${ }^{3}$ LP is an indicator of chronic sarcoidosis. There is a $50 \%$ risk of developing LP if the patient has upper res- piratory tract sarcoidosis, although each can present separately. ${ }^{4}$ Cutaneous sarcoidosis can be the presenting sign of systemic sarcoidosis, and work-up is indicated in all patients with cutaneous manifestations.

Annual incidence is thought to be between 1 and 64 per 100,000 . It is more common among some populations of African origin, women and ages between 45 and 65.,5

The cause of cutaneous sarcoidosis is unknown, although some theories exist. In literature, it has been proposed that it could be due to the unusual host reaction to Mycobacterium paratuberculosis, fungi, histoplasmosis or other infective agents. Other studies refer to it as a cell mediated immune response were granulomas are formed as a result of activation of CD4 T lymphocytes and macrophages which release cytokines. ${ }^{3,5}$ Sarcoidal inflammation has been related to multiple factors, including infectious antigens, genetics and environmental triggers, and the immune system. The immune system is thought to be related in that it involves Th1 predominant response with interferon-gamma and tumor necrosis factor. In addition, there is evidence in literature suggesting a role of Th17 inflammation and interplay with the innate immune system. ${ }^{5}$

The histopathological changes are similar in all organs affected by sarcoidosis. The gold standard feature in histology is noncaseating granuloma with sparse lymphocytic component known as naked granulo$m a$. The granulomas are usually observed in the dermis but can extend in some cases to the subcutaneous tissue. At the center of the granuloma, CD helper cells or induced $\mathrm{T}$ lymphocytes are present, whereas at the periphery small proportion of CD8 suppressor and cytotoxic $\mathrm{T}$ lymphocytes can be present. Granulomas can contain few or no giant cells. ${ }^{6}$

LP rarely resolves spontaneously and may cause cosmetic disfigurement, nasal obstruction and fibrotic pulmonary complications due to extensive involvement of the nasal cavity and maxillary sinus. ${ }^{7}$

In this paper, we report a case of systemic sarcoidosis presenting with rare subtype of LP. We reviewed the available literature, and explained the significant negative impact the condition could have on the patients' lives despite it being non-life threatening. In addition, our manuscript creates further ground for future studies in LP management and treatment approaches.

\section{Case Report}

A 55-year-old Caucasian woman known
Correspondence: Amal Hubail, Hospital of Skin and Venereal Disease Hospital names after VA Rakhmanov. I.M. Sechenov First Moscow State Medical University, Bolshaya Pirogovskaya street 4c1, Moscow, Russia. Tel.: +79857598534 .

E-mail: dr.amalhubail@gmail.com

Key words: Sarcoidosis; Cutaneous sarcoidosis; Lupus pernio; Nasal sarcoidosis.

Contributions: the authors contributed equally.

Conflict of interest: the authors declare no potential conflict of interest.

Funding: none.

Received for publication: 28 March 2018.

Revision received: 30 June 2018.

Accepted for publication: 21 July 2018.

This work is licensed under a Creative Commons Attribution-NonCommercial 4.0 International License (CC BY-NC 4.0).

CC Copyright A. Hubail et al., 2018

Licensee PAGEPress, Italy

Dermatology Reports 2018; 10:7696

doi:10.4081/dr.2018.7696

case of stage II sarcoidosis presented complaining of the enlargement of her nose since 2 years. The patient denied any systemic symptoms respiratory, cardiovascular, neurological, ocular, or musculoskeletal symptoms.

In 1998 , at the age of 37 , a chest X-ray showed changes in the patient's lungs. The diagnosis of stage II sarcoidosis was established on the basis of clinical symptom and radiological findings on chest xray which was supported by histological evidence showing noncaseating epithelioid-cell granulomas in biopsy. In the course of a month, the patient was treated with prednisolone, chloroquine and vitamin E. In 2004, she had a flare up of her disease with pronounced dyspnea, $15 \mathrm{~kg}$ weight loss within few years, general fatigue and joints swelling and pain especially in the upper limb. The treatment at this time included budesonide, formoterol, prednisolone, ipratropium bromide, and ambroxol hydrochloride.

On presenting examination, the patient had well demarcated violet red lesions on the skin of the nose up to $4 \mathrm{~cm}$ in diameter, with visible telangiectasia. The nose was visibly enlarged, with thickening of the ala and septum of the nose. She had difficulty in nasal breathing, dyspnea at rest and wheezing (Figures 1-3).

The following comorbid conditions were identified: coronary artery disease, atherosclerotic cardio sclerosis, mild mitral valve 
prolapse (grade 1) and bullous emphysema.

The complete blood count, clinical urine analysis, biochemical blood tests and electrolytes especially calcium were within normal limits Serum angiotensin-converting enzyme was elevated around twice the normal range and Kveim-Siltzbach skin test positive.

Histopathology of a biopsy from the skin of the nose showed epithelioid granulomas with scarce amount of lymphocytes present on the periphery and single giant multinucleated cells. Caseation necrosis was absent. The vessels of the superficial vascular plexus had a dilating lumen. There were disseminated, distinct, non-caseating naked granulomas (i.e. they lacked lymphocytes in the periphery). In addition, granulomas entailed giant cells; it spared the dermal epidermal junction and adnexa. Ziehl Neelsen stain showed no acid fast bacilli organism (Figure 4).

Digital two-dimensional X-ray of the chest wall showed a significant heterogeneous induration of the lung tissue in the perihilar parts of the both lungs. The right lung was reduced in size. A limited accumulation of air without distinct contours was noted in the supra-diaphragmatic parts. The lung hila were consolidated, and lymph nodes were significantly enlarged on both sides. The follow-up Computed Tomography series from January 2015 showed almost the same level of disease. Radiographs of the hands and feet showed an uneven joint space narrowing in the interphalangeal joints, regional osteoporosis and moderately expressed arthritic changes.

The patient was consulted by otolaryngologist that concluded that the patient had difficulty in nasal breathing. The shape of the outer part of the nose was significantly changed due to the noseband widening, with thickening of the ala. The nasal mucous membranes were moderately hyperplastic. The posterior pharyngeal wall was within normal limits. Nasal bone and sinus radiographs results were not available to us and nasal mucosal biopsy was not performed.

After establishing the diagnosis of LP, the patient was treated with: i) Oral prednisolone, starting at $30 \mathrm{mg}$ daily and to be tapered over 2-4 months depending on the response (Table 1); ii) Topical cream clobetasol dipropionate $0.05 \%$ twice daily until observation of clinical improvement; iii) Sun protection all year round with SPF > 50.

In the course of the treatment, present lesions were flattening, the intensity of color diminished, the nose reduced in size, nasal breathing was restored, and dyspnea and wheezing were absent. The patient was observed to have significant improvement and was recommended to reduce the dose of prednisolone gradually to a permanent discontinuation under the supervision of the dermatologist. Her prognosis is thought to be variable, chronic state with exacerbation and remissions mainly due to the systemic sarcoidosis. One year follow up shows marked but not complete improvement (Figure 5).

\section{Discussion}

Sarcoidosis has nonspecific clinical manifestations that might correlate with lesions in any organ, ${ }^{7}$ however it is often extrapulmonary manifestations that lead to the diagnosis. ${ }^{6}$ Skin changes in sarcoidosis occur with a frequency of $25 \%$ to $56 \%{ }^{6}$

LP is a type of cutaneous sarcoidosis that can be related to chronic fibrotic sarcoidosis, hyperglobulinemia and hypercalcemia. Generally, LP is a chronic disease that is non-life threatening but it can result in facial disfigurement which in turn leads to emotional instability and may affect the quality of life of the patient.

In 1869 sarcoidosis was first described as a disease of the skin (papillary psoriasis) by J. Hutchinson, followed by Besnier in 1889 , and in Boeck in 1899. It was not until the middle of the twentieth century that the term sarcoidosis was used. ${ }^{1,8}$ The affected skin of the nose turns red, purple or violet because of the large number of vessels in the affected area. The disease is chronic, usually relapse in winter. Sarcoidosis is called the great imitator great mimicker due to the nonspecific clinical manifestations, which might correlate with a lesion of any organ, however it is often extrapulmonary

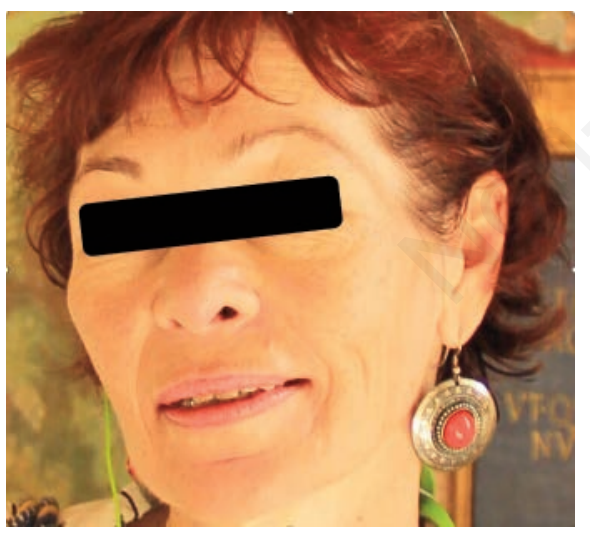

Figure 1. Patient's appearance before diagnosis of Lupus pernio.
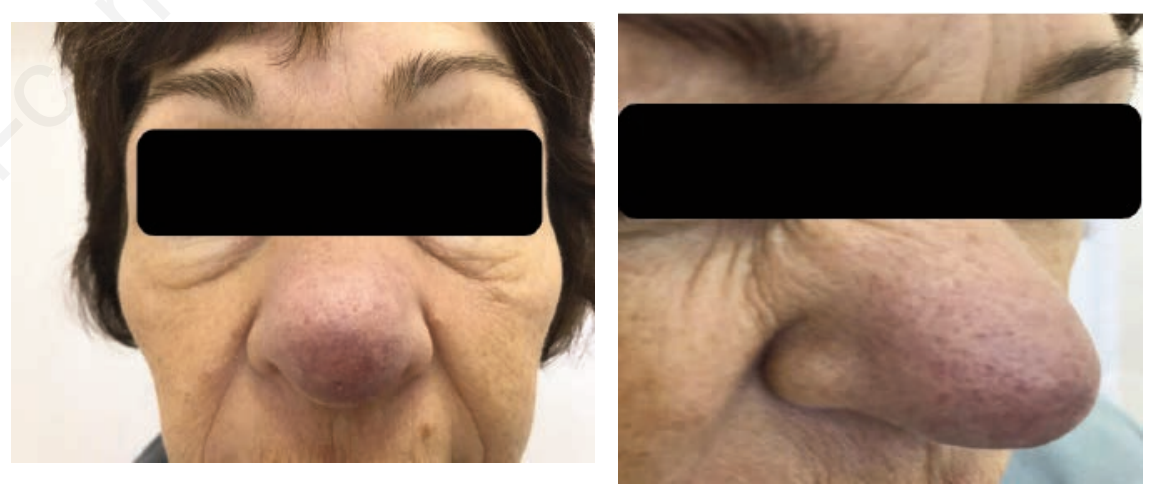

Figure 2. Patient's appearance after diagnosis of Lupus pernio.
Figure 3. Patient's appearance after diagnosis of Lupus pernio.

Table 1. Treatment with oral prednisolone, starting at $30 \mathrm{mg}$ daily and to be tapered over $2-4$ months depending on the response.

\begin{tabular}{lcccccccc} 
& Monday & Tuesday & Wednesday & Thursday & Friday & \multicolumn{2}{c}{ Saturday } \\
Week 1 & $15 \mathrm{mg}$ & $15 \mathrm{mg}$ & $15 \mathrm{mg}$ & $15 \mathrm{mg}$ & $14 \mathrm{mg}$ & $15 \mathrm{mg}$ & $15 \mathrm{mg}$ \\
Week 2 & $15 \mathrm{mg}$ & $15 \mathrm{mg}$ & $14 \mathrm{mg}$ & $15 \mathrm{mg}$ & $14 \mathrm{mg}$ & $15 \mathrm{mg}$ & $15 \mathrm{mg}$ \\
\hline Week 3 & $15 \mathrm{mg}$ & $15 \mathrm{mg}$ & $14 \mathrm{mg}$ & $15 \mathrm{mg}$ & $14 \mathrm{mg}$ & $15 \mathrm{mg}$ & $14 \mathrm{mg}$ \\
Week 4 & $14 \mathrm{mg}$ & $15 \mathrm{mg}$ & $14 \mathrm{mg}$ & $15 \mathrm{mg}$ & $14 \mathrm{mg}$ & $15 \mathrm{mg}$ & $14 \mathrm{mg}$ \\
\hline
\end{tabular}


manifestations that lead to the diagnosis. Clinical manifestations of the disease are related to the methods and timing of detection. Skin changes in sarcoidosis occur with a frequency of from $25 \%$ to $56 \%$, which gives high importance to a thorough examination of the skin. Rare manifestations include lichenoid or psoriasiform lesions, ulcers, angiolymphoid, ichthyosis, alopecia, hypopigmentation spots, loss of nails, and subcutaneous sarcoidosis. Sarcoidosis can also manifest as annular, indurative plaques with no granuloma..$^{5,9}$

Sarcoidosis of the skin can present in various forms: i) Typical form: skin sarcoid Beck (cutaneous sarcoidosis Becca (large nodular, small nodular, and diffuse-infiltrative); LP (Besnier-Tenneson syndrome), angiolupoid Brocq Pautrier; subcutaneous sarcoidosis Darier Roussy; ii) Atypical forms: spotted, lichenoid, psoriasiform sarcoidosis; iii) Mixed form: finely nodular and coarsely knotted, finely nodular and subcutaneous, finely nodular and angiolymphoid, diffuse-infiltrative and subcutaneous.

\section{Investigations and differential diag- nostics of Lupus pernio}

The diagnosis is based on the results of clinical and histological studies. A biopsy is taken from the affected skin. Histologically, LP is characterized by the presence of naked epithelial cell granulomas that are, without inflammatory reaction around and inside the granuloma, without caseosa (fib- rinoid necrosis); presence of different numbers of giant cells of type of PirogovLanghans type and foreign body unchanged or atrophic epidermis.

Laboratory investigations usually show no significant changes, except for hypercalceamia, serum angiotensin converting enzyme level $>2-3$ times the upper limit of normal, and Kveim-Siltzbach skin test positive in about $91 \%$ of patients with LP, compared with a positivity of $65 \%$ o in sarcoidosis overall. The main radiological manifestation of pulmonary sarcoidosis is bilateral increase of intrathoracic lymph nodes. Enlarged lymph nodes are clear of polycyclic outlines. In the early stages of the disease, there is increased pulmonary pattern due to peribronchial and perivascular mesh. Later there is development of focal shadows (original marmoristeria) of various size, round shape, two-way, spread in all lung fields, but predominant in the lower and middle parts of the lungs. With the progression of the process, there is an intense proliferation of connective tissue diffuse pneumosclerosis changes (honeycomb lung). X-rays may reveal bone destruction in the form of multiple foci rarefaction of bone tissue, mainly in the phalanges of hands and feet. In this form of limited emptiness round or oval, clearly seen in x-rays is so-called custody osteitis, symptom of osteitis Morozova Yunglinga. More rarely, bones of the skull and long bones are involved in the process. The eye may be affected by sarcoidosis mainly the anterior and posterior uveitis (the most common form of disease), conjunctivitis, corneal opacity, cataracts or glaucoma, changes of the iris, or decreased visual acuity.

Differential diagnosis includes the following: malignant and benign tumors (lymphoma and cutaneous pseudolymphoma, benign, Kaposi's sarcoma, mastocytosis, melanoma, histiocytoma, etc.), granuloma faciale, specific infections (leprosy, skin tuberculosis, and leishmaniasis), cutaneous manifestations of metabolic disorders (xanthomas, necrobiosis lipoidica, etc.), lupus erythematosus, lichen planusand others. ${ }^{10}$

\section{Treatment}

Cutaneous sarcoidosis requires treatment of patients who are symptomatic, with progressive disease or cosmetic disfigurement. Treatment is required in LP due to the risk of disease progression or cosmetic disfigurement implying physical, psychological and social impairment that may be acquired. Nevertheless, treatment is difficult and complicated due to the unpredictable course of the condition. ${ }^{11}$

Numerous therapeutic options are available in literature on the treatment of sarcoidosis but few randomized trials are available addressing the treatment options of various cutaneous manifestations and no treatment for sarcoidosis is yet approved by the United States Food and Drug Administration. The first line therapy is local then systemic glucocorticosteroid. For limited skin involvement, intralesional

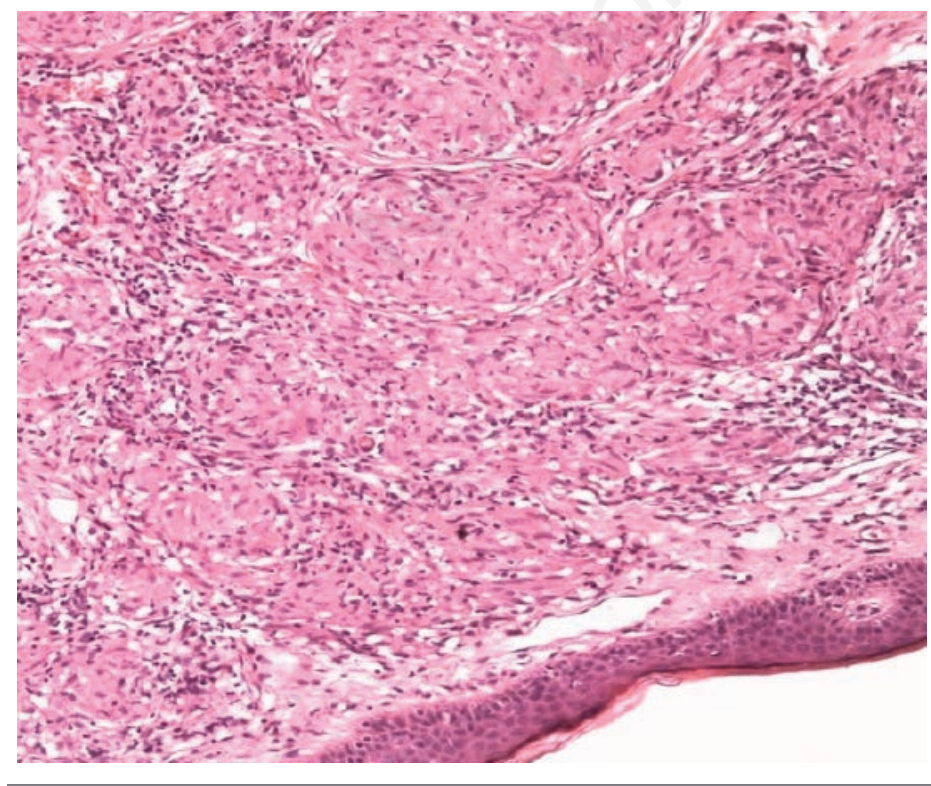

Figure 4. Histology result of the patient's biopsy. Epithelioid granulomas with lymphocytes present on the periphery. Caseation necrosis absent. The vessels of the superficial vascular plexus have a dilating lumen. Disseminated, distinct, non-caseating naked granulomas visible.

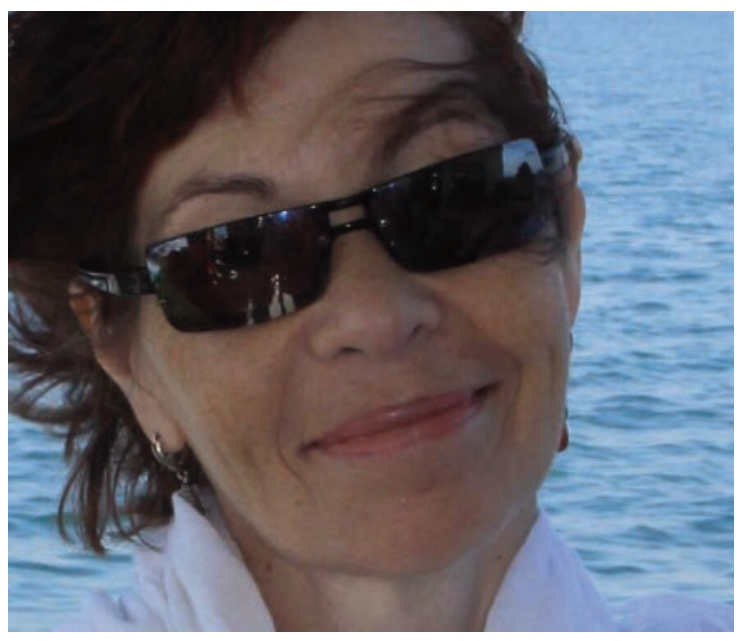

Figure 5. Patient's appearance on 1 year follow up. 
injections of triamcinolone acetonide is used. Several other management modalities which are given in literature include chloroquine, hydroxychloroquine, methotrexate, azathioprine, cyclophosphamide, thalidomide, and infliximab. ${ }^{11,12}$

LP in all cases requires treatment due to its destructive nature. Despite the lack of evidence for treatment of LP, topical corticosteroid therapy has shown favorable responses. Systemic corticosteroids are used for thick plaques unresponsive to topical corticosteroids and where intralesional corticosteroids would be impractical and painful. ${ }^{11}$ Systemic glucocorticosteroids (prednisolone $30-40 \mathrm{mg}$ per day) has been associated with great results and almost complete resolution. Intralesional therapy also proved better than local therapy in such cases. Antimalarials, methotrexate and tetracycline derivatives are glucocorticoidsparing agents that are used either as alternatives or in order to decrease the dose of corticosteroid. ${ }^{11}$

LP may fail to respond to the above and disease modifying drugs may be of benefit. Infliximab used as monotherapy or combination has been shown in some trials to be superior in the treatment of LP, which is administered IV 3 to $5 \mathrm{mg} / \mathrm{kg}$ initially then subsequent doses at 2, 4,6,10 and 14 weeks. ${ }^{13}$ Other studies showed that halobetasol propionate $0.05 \%$ ointment resulted in significant flattening of the lesions and is given twice a day for approximately 10 weeks. ${ }^{14,15}$

Another drug which has shown good results in a case studied is Lenalidomide $10 \mathrm{mg}$ daily for approximately 3 months. ${ }^{16}$ Some research articles have concluded with promising results using either pulsed dye laser or photodynamic therapy. ${ }^{16-18}$ Lastly, minocycline has been shown to have good results but needs more research to prove its efficacy. ${ }^{19}$

The strengths of the diagnostic and therapeutic approach in this case were that the patient accepted a biopsy sample to be taken from the face which provided an ultimate confirmation of the diagnosis and treatment was immediately started. Another strength was that the patient was already diagnosed with systemic sarcoidosis which made cutaneous sarcoidosis the first thing to come in mind. The limitations of the case are that the patient has underlying systemic sarcoidosis and other pathologies, that require continuous follow up and special care when prescribing medications to avoid cross reactions and adverse effects.

\section{Conclusions}

LP follows a chronic course and can persist for several years (2-25 years). Lung and cardiac function should be monitored. Lesions of more than 2 years tend to persist and present active chronic disease. . $^{3,5}$

In conclusion, LP is an uncommon presentation of a systemic illness, were early recognition is important in order to avoid a delay in treatment and worsening prognosis. Although non-life threatening, it has been shown to affect the quality of life of the patients affected. Having a patient with systemic sclerosis developing cutaneous manifestation gives us the opportunity to think of how to treat both the condition in hand along with the main systemic illness.

Clinical observation and awareness along with essential investigations can help to diagnose LP. The challenge encountered in practice is to the management approach. In future further research has to be done to reach an evidence basedmanagement for patients with LP as literature is lacking concrete evidence to support treatment.

\section{References}

1. Gopikrishnan A, Rita V. Lupus pernio without systemic involvement. Indian Dermatol Online J 2013;4:314-7.

2. Scadding JG, Mitchell DN. Sarcoidosis. London, UK: Eyre \& Spottiswoode; 1967.

3. Howard A. Non-infectious granulomas. In: Bolognia J, ed. Textbook Dermatology. Vol 2. London, UK: Mosby; 2008.

4. Wanat AK, Rosenbach M. Cutanous Sarcoidosis. Clin Chest Med 2015;36:685-702.

5. Gawkrodger DJ. Sarcoidosis. In: Burns T, Breathnach S, Cox N, Griffiths C, eds. Rooks Textbook of Dermatology. Vol. 5. Massachusetts: Blackwell Science Ltd; 2016. pp 98.1-98.13.

6. Neville E, Mills RG, Jash DK, et al. Sarcoidosis of the upper respiratory tract and its association with lupus pernio. Thorax 1976;31:660-4.

7. Amor K. Plaques and papules on the nose. Am Fam Physician 2006;73:14312.

8. Paller A, Gilcrest Bam Leffell DJ, et al. Fitzpatrick dermatology in general medicine. London; McGraw Hill Medical; 2012.

9. Fernandez Faith, McDonnell J. Cutaneous Sarcoidosis: differential diagnosis. Clin Dermatol 2007;25:27687.

10. Peter S, Kim M, Maor S et al. Pulmonary arterial hypertension. Clin Chest Med 2013;34:799-857.

11. Baughman RP, Lower EE. Evidencebased therapy for cutaneous sarcoidosis. Clin Dermatol 2007;25:334.

12. Anjaneyan G, Vora R. Lupus pernio without systemic involvement. Indian Dermatol Online J 2013;4:314-7.

13. Stagaki E, Mountford WK, Lackland DT, et al. The treatment of lupus pernio: results of 116 treatment courses in 54 patients. Chest 2009;135:468-76.

14. Khatri KA, Chotzen VA, Burrall BA. Lupus pernio: successful treatment with potent topical corticosteroid. Arch Dermatol 1995;131:617-8.

15. Dalm VA, van Hagen PM. Efficacy of lenalidomide in refractory lupus pernio. JAMA Dermatol 2013;149:493-4.

16. Ashinoff R, Dosik JS. Treating lupus pernio with the $585 \mathrm{~nm}$ pulsed dye laser. Skin Aging 1999; 7:93.

17. Roos S, Raulin C, Ockenfels HM, et al. Successful treatment of cutaneous sarcoidosis lesions with the flashlamp pumped pulsed dye laser: a case report. Dermatol Surg 2009;35:1139-40.

18. Holzmann RD, Astner S, Forschner T, et al. Scar sarcoidosis in a child: case report of successful treatment with the pulsed dye laser. Dermatol Surg 2008;34:393-6.

19. Bachelez H, Senet P, Cadranel J, et al. The use of tetracyclines for the treatment of sarcoidosis. Arch Dermatol 2001;137:6973. 